\section{Interstellar dustmen's convention}

from Ant. Whitworth

A workshop on Interstellar Grains was organised by University College Cardiff at Gregynog Hall on July 11-13.

DusT gets into everything, or so they always say, and certainly it seems the cosmos is no exception. In astrophysics, dust grains (sub-micron sized particles constituted of compounds of $\mathrm{Si}, \mathrm{C}, \mathrm{N}, \mathrm{O}, \mathrm{Fe}, \mathrm{Mg}$ ) are continually invoked, and invested with new capabilities, to explain puzzling observations. Yet the recent increase in constraints on dust models has not resulted in a more definite picture of dust grains, but rather in the introduction of more free parameters (such as multi-component models and localised variations). Nevertheless, a pattern emerges, and with it the realisation that grains have many very fundamental roles in the cosmos.

At the recent workshop $\mathrm{H}$. C. van de Hulst (Sterrewacht, Leiden) stressed that the interstellar medium is a very hostile environment for grains; and J. M. Greenberg (Sterrewacht, Leiden) pointed out the need to consider how intense irradiation may modify grain properties. J. P. Bibring (Laboratoire René Bernas, Orsay) has investigated grains in lunar and meteoritic samples, and concluded that irradiation of grains in circumstellar environmentssay by stellar winds, energetic photons, or cosmic rays during their formation -may significantly round the grain and amorphise its internal structure. D. R. Huffman (University of Arizona, Tucson) pointed out that this bears on the identification of the $10 \mu \mathrm{m}$ feature in infrared spectra which requires amorphous silicates; whereas the $2,200 \AA$ ultraviolet feature requires wellordered graphite. S. Ramadurai (Institute of Astronomy, Cambridge) argued that carbonaceous chondrites contain interstellar graphite grains from the protosolar nebula, as evidenced by their anomalously high boron content, which is attributed to cosmic ray bombardment of graphitic carbon.

But a cogent case was made by N. C. Wickramasinghe (University College, Cardiff) for considering grain materials with a volatility intermediate between the very volatile 'dirty ices' (hydrides of $\mathrm{C}, \mathrm{N}$ and $\mathrm{O}$ ), and the more refractory silicates and graphite. $\mathrm{He}$ cited organic polymers, and in particular suggested that conditions in dark clouds may favour condensation of the widespread formaldehyde mole- cule into elongated grains of polyoxymethylene (POM). There remains outstanding the problem of producing sufficient formaldehyde, but there is already good evidence in support of the general thesis. D. A. Mendis (University of California, La Jolla) reported on the physical parameters for the grains producing 10 and $18 \mu \mathrm{m}$ features in comets, and indicating an evaporation temperature $\sim 500 \mathrm{~K}$ : this rules out silicates, but is very compatible with many polymers, including POM. B. Thomas and co-workers (University of Wales Institute of Science and Technology, Cardiff) have measured strong absorption bands around 10 and $18 \mu \mathrm{m}$ in thin films of POM.

M. J. Barlow (Sussex University) proposed a resolution of the problem of supplying sufficient refractory grains to the interstellar medium: refractory grains condense in almost every stellar mass loss situation, and are probably only destroyed by direct involvement in star formation after a mean lifetime $\gtrsim 10^{\circ}$ yr. D. P. Gilra (University College, Cardiff) deduced the existence of a temperature minimum in the atmospheres of late $\mathrm{N}$-type carbon stars where $\mathrm{SiC}_{2}$ grains are condensing. C. D. Andriesse (Sterrewacht, Roden) presented infrared observations of $\eta$ Car as evidence of dust suddenly condensing in the mass outflow at a radius $\sim 0.03$ pc. H. Okuda (Kyoto University) interpreted polarisation data on VY Can Maj in terms of a circumstellar dust disk: is this disk protoplanetary? J. Silk (University of California, Berkeley) prescribed a scenario for fragmenting and reconstituting dust in cocoons around newly formed massive stars.

W. W. Duley (York University, Ontario) offered new hope for identifying the diffuse bands: he attributed narrow lines to impurities in the bulk grain material, and the adjacent broader features to the same impurities in grains which are so small that their lattice constant is altered. D. P. Gilra (University College, Cardiff) calculated infrared absorption cross sections for small ellipsoidal particles, taking account of surface modes, and stressed the great importance of grain shape. A. P. Whitworth (University College, Cardiff) argued that in neutral clouds dielectric grains are charged stochastically on a time scale $\sim 10^{3} \mathrm{~s}$, typically having $0, \pm 1$ electron charges. Conséquently, grains are effectively untied from the magnetic field, can move through the gas under gravity and/or radiation pressure, and can be aligned in the required sense. P. A. Aannestad (University of Arizona, Tucson) suggested that stochastic grain charging may also enable grains to deplete $\mathrm{C}^{+}$, $\mathrm{N}^{0}$ and $\mathrm{O}^{\circ}$ equally, as inferred from observation. But D. A. Williams (Uni- versity of Manchester Institute of Science and Technology) concluded that the depletion of CNO may anyway be negligible.

A number of interesting roles have been suggested for grains in cosmology. A. J. R. Prentice (University of Oxford) argued that in the rotating protosolar cloud, grains fall to the centre creating a core sufficiently massive to stabilise the rest of the cloud, and establishing composition gradients which will explain the solar neutrino deficiency. I. P. Williams (Queen Mary College, London) has shown the basic part which grains may play in forming the planets and determining their different compositions. M. Rowan-Robinson (Queen Mary College, London) poses the question : is dust always associated with violent events in galactic nuclei? One might also ask whether such events are not a major source of the dust in the cosmos.

\section{Galaxy formation}

from Janet $E$. Jones

A workshop meeting on "The Formation and Evolution of Galaxies" was held at the Institute of Astronomy, Cambridge, on August 4-8, and supported by the Gravity Research Foundation.

LEAVING aside the still controversial topic of the origin of pre-recombination density inhomogeneities, the emphasis at the workshop was focused on later epochs, with theories for early galactic evolution and observational "clues" on the topic being well discussed. The workshop opened with a number of papers on the covariance and multiplicity functions, the purpose being to use the presently observed spatial distribution and luminosity functions of galaxies as indicators of the primordial density fluctuation spectrum.

It was pointed out that non-linearities would tend to increase the ultimate slope of the covariance function, thus making it more difficult for an initially flat, $n=0$ (Poisson), density fluctuation spectrum to account for the observed covariance function: an $n=-1$ initial spectrum seems to give a better fit (J. R. Gott and M. Rees, Institute of Astronomy, Cambridge). Moreover, the slope of the covariance function seems to depend on galaxy type, being steeper for ellipticals than for spirals (M. J. Geller, Harvard University). This could suggest some difference in the primordial density fluctuation spectrum leading to the two types, although later attempts to model galactic evolution did not incorporate this property. In- 